\title{
Designing a Conceptual Model for the Relationship Between Shift Work, Job Stress, Job Satisfaction and Health: A Case Study in Petrochemical Industry
}

\author{
Fatemeh Zameni ${ }^{1}$, Ahmad Soltanzadeh ${ }^{2 *}$, Parvin Nasiri ${ }^{3}$
}

1. MSc Student, Department of Environmental Management (HSE), Science and Research Branch, Islamic Azad University, Tehran, Iran

2. Assistant Professor, Department of Occupational Safety \& Hygiene Engineering, Faculty of Health and Research Center for Environmental Pollutants, Qom University of Medical Sciences, Qom, Iran

3. Professor, Department of Occupational Hygiene Engineering, School of Public Health, Tehran University of Medical Sciences, Tehran, Iran

\begin{tabular}{ll}
\hline \multicolumn{2}{c}{ Article Info } \\
\hline Original Article \\
Received: & $2018 / 09 / 15$ \\
Accepted: & $2018 / 10 / 31$ \\
Published Online: & $2018 / 10 / 31$
\end{tabular}

DOI: 10.30699/jegon.6.2.64

Use your device to scan and read the article online

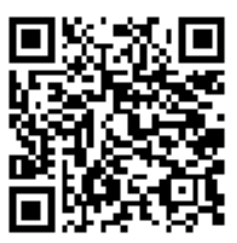

Corresponding Information

\section{Ahmad Soltanzadeh}

Assistant Professor, Department of Occupational Safety \& Hygiene Engineering, Faculty of Health and Research Center for Environmental Pollutants,

Qom University of Medical Sciences, Qom, Iran Email:

soltanzadeh.ahmad@gmail.com

\section{Abstract}

Introduction: In the modern industrial world, the health of the employees in different Occupations can be influenced by the interaction of various risk factors. So, the purpose of present study was to assess and analyze the relationship between the factors including shift work, job stress, job satisfaction and health in a petrochemical industry.

Materials \& Methods: The present study was conducted using analytical and cross-sectional method. The statistical population was all employees of a petrochemical company (4000 people). Using the Cochran formula, a sample of 360 individuals were selected using simple random sampling method. Data was collected using a high reliability integrated questionnaire (Cronbach's alpha $=0.83$ ). Data was analyzed using SPSS22.0.

Results: The analytical results of relationship between shift work, occupational stress, occupational satisfaction and health showed that all hypotheses in the conceptual model were approved and there was a significant relationship between these factors $(P<0.001)$. Also, the results showed that, in addition to the direct effect of shift work on health, due to the effect on occupational stress and occupational satisfaction, it was indirectly related to health $(P<0.001)$.

Conclusion: The findings indicated that shift work as a direct factor and as a mediator and indirect factor through impact on factors such as occupational satisfaction and stress can affect the health of employees. Therefore, considering this organizational phenomenon and its redesign or its change can play an important role in increasing the level of occupational health and motivation as well as organizational productivity.

Keywords: Shift work, Health, Occupational stress, Occupational satisfaction, Petrochemical Industry 


\section{مقالة يثوهشى \\ طراحى مدل مفهومى ارتباط نوبت كارى، استرس شغلى، رضايت شغلى و سلامتى مطالعهُ موردى در صنعت گيتروشيمى}

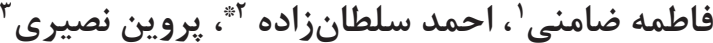

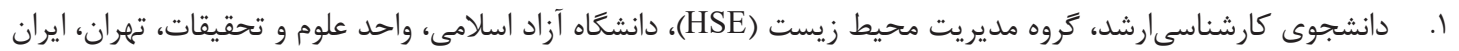

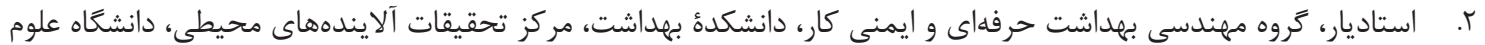
يزمكى قمه، قم، ايران

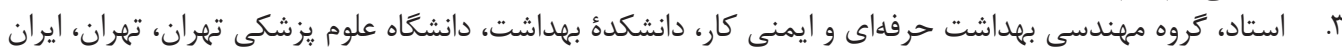

\begin{tabular}{|c|}
\hline جكيد \\
\hline 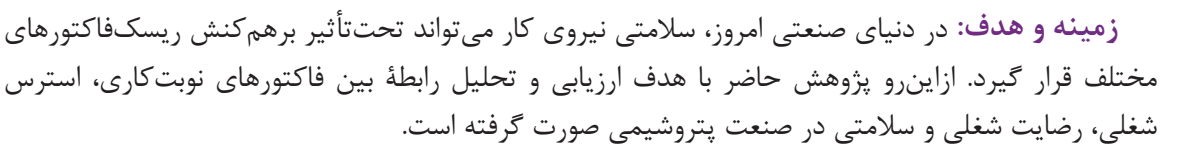 \\
\hline 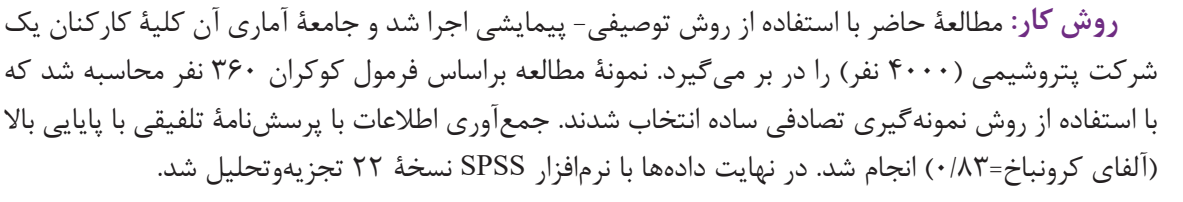 \\
\hline 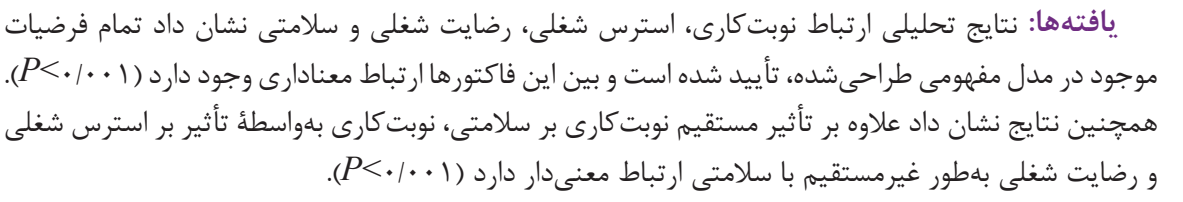 \\
\hline 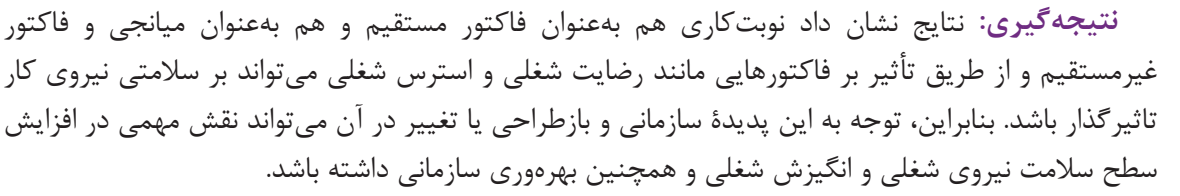 \\
\hline
\end{tabular}

اطلاعات مقاله

تاريخ وصول:

نويسندهُ مسئول:

احمد سلطانزاده

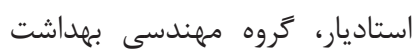

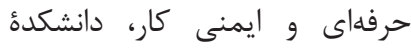
بهداشت، مركز تحقيقات آلايندههاى لمراى

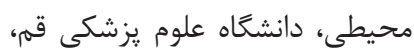

قم، ايران

يست الكترونيك: soltanzadeh.ahmad@gmail.com
عضلانى شود [C،V]. مطالعات Choobineh و همكاران نشان

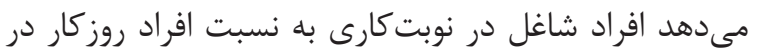

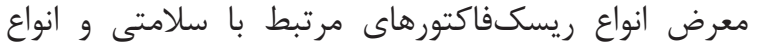

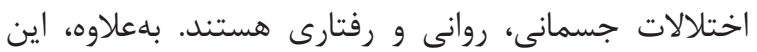

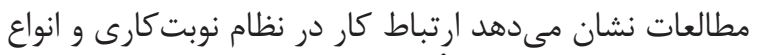

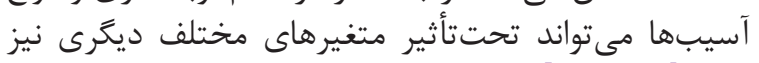

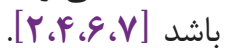

رضايت شغلى يكى از عوامل بسيار مهرم در موفقيت

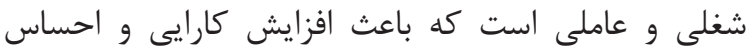

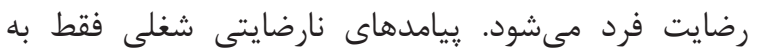

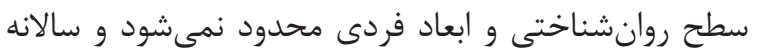

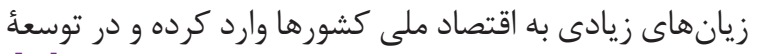

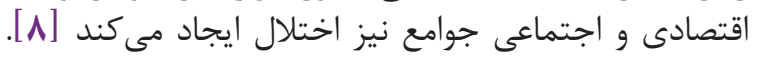

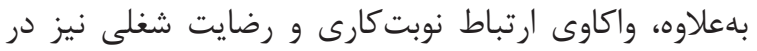

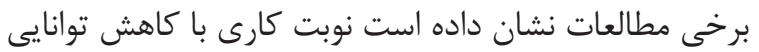

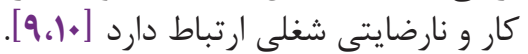
استرس شغلى ياسخهاى هيجانى آزاردهندهاى است كه در

\section{مقدمه}

امروزه نوبت كارى بلهعنوان عاملى مؤثر در سلامت افراد

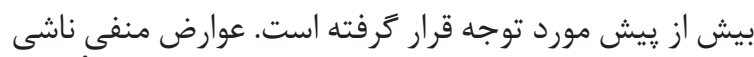

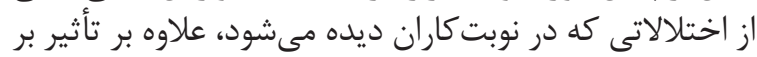

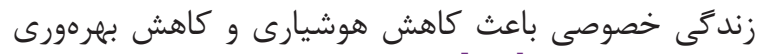

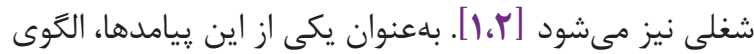

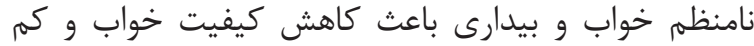

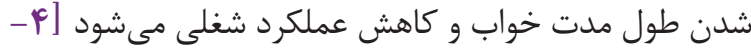

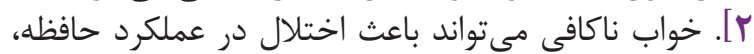

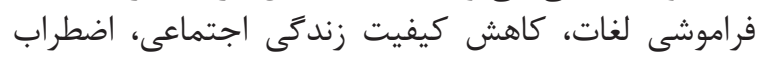

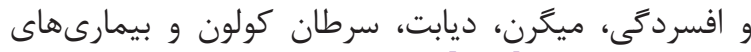
قلبى -عروقى شود [ه، هـ]

مطالعات مختلفى اثرات مستقيم و ميانجى نوبت كارى

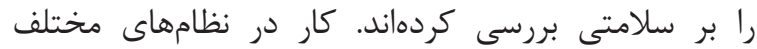

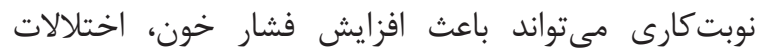

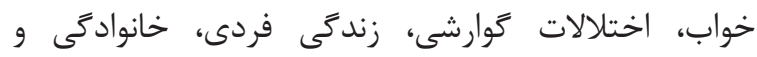
اجتماعى، اختلالات روحى -روانى، قلبى -عروقى و اسكلتى - 
آمارى براساس فرمول كوكران · عس نفر بوده است. انتخاب

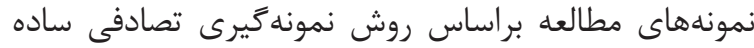

$$
\text { صورت گرفت. }
$$

ابزار گردآروى اطلاعات در اين مطالعه، يرسشنامئ

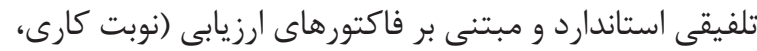

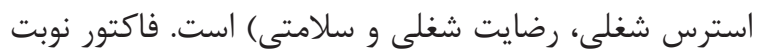

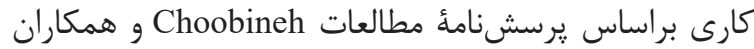

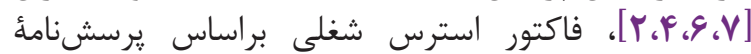

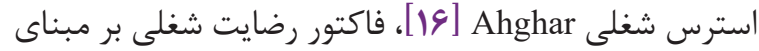

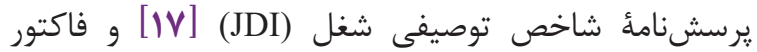

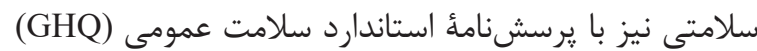

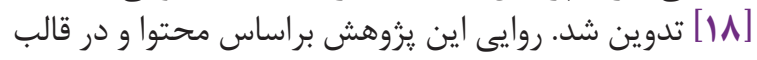

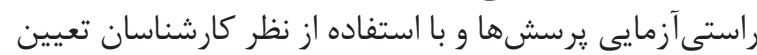

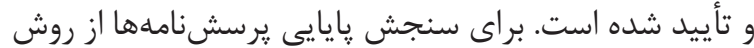

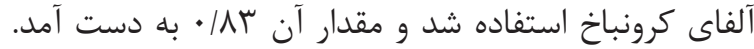

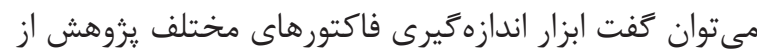

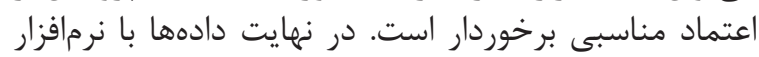
نسخئ r TPSS

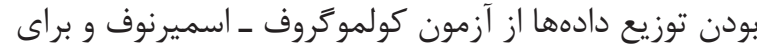

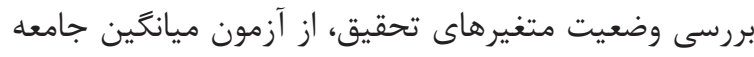

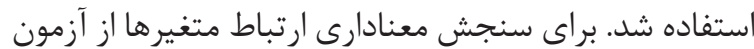

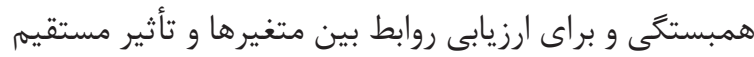

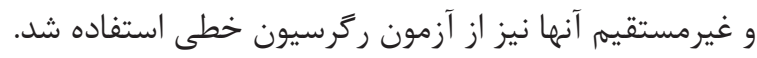

\section{مدل مفههومى مطالعه}

مدل مفهومى مطالعه در شكل شماره ا آمده است.
صورت نبودِ تناسب بين خواستهان و الزامات شغلى، منابع يا

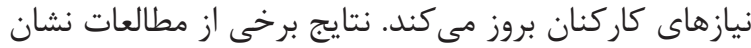

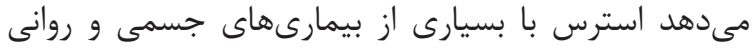

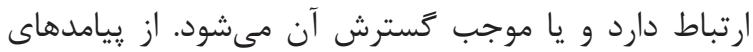

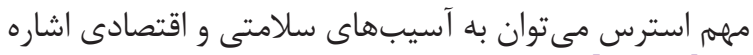

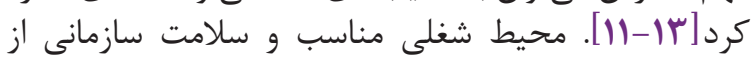

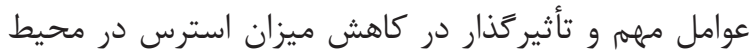

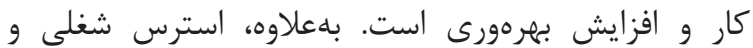

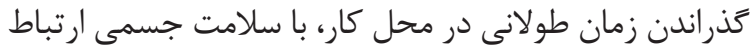

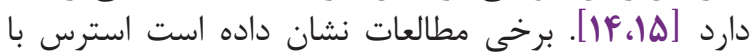

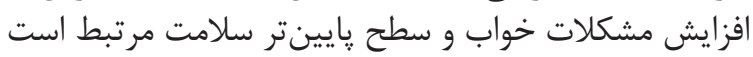

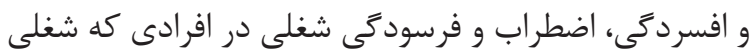

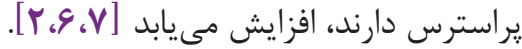

براساس آنجه بيان شد، اثرات تعاملى و مت متقابل ريسكفاكتورهاى مختلف مانند كار در نظام نوبت كاتئ كارى و و

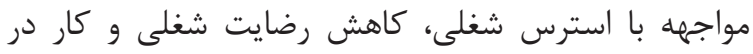

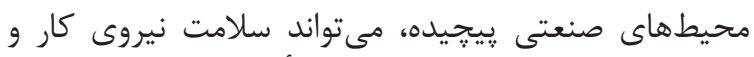

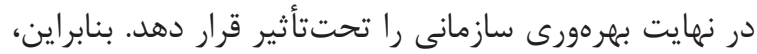

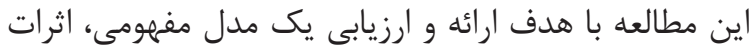

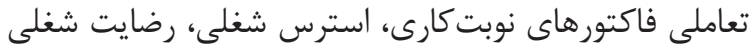
و سلامتى را در صنعت يتروشيمى بررسى كرده است نوست

$$
\text { روش كار }
$$

مطالعه حاضر با استفاده از روش توصيفى - يّمايشى اجرا

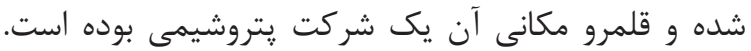

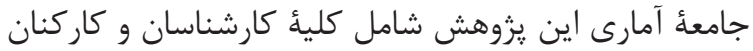

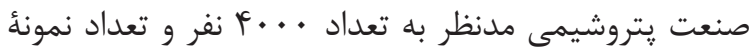

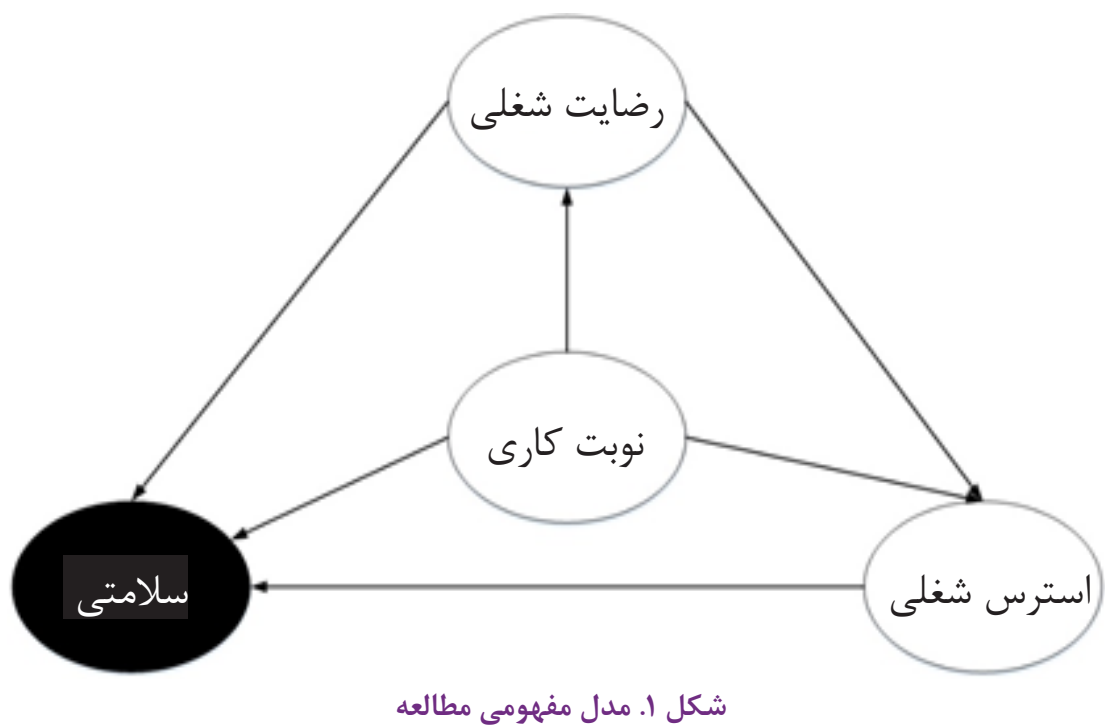

تحصيلات كارشناسى و بالاتر داشتند.

بافته ها

نتايج ارزيابى دادههاى مطالعه براساس آزمون كولموكروف-

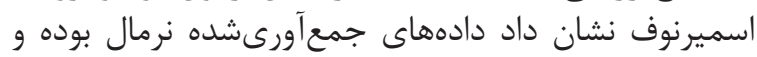

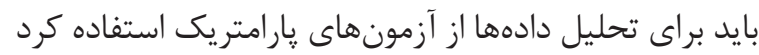

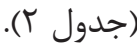

نتايج فردى و دموگرافيك افراد مطالعهشده در جدول

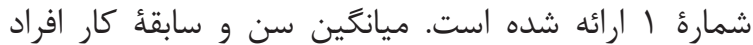

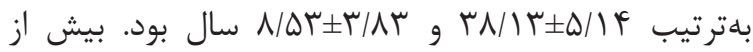

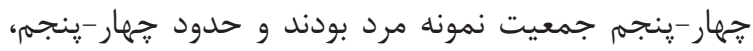


بر سلامتى تأثير كذار است و بزركى اين تأثير بهخوبى ارزيابى )

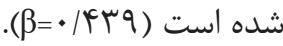

ارزيابى مدل مفهومى ارائدشده براساس فاكتور رضايت

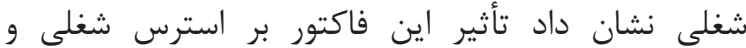

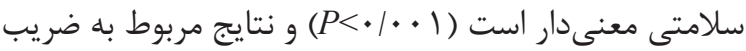

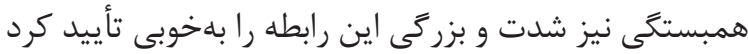

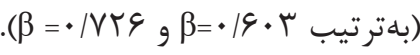

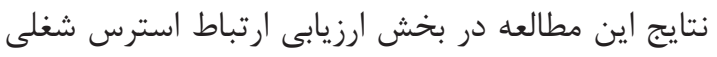

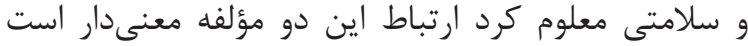

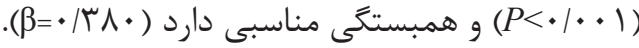

مدل مفهومى اين مطالعه براساس شكل شمارء ب ارائه

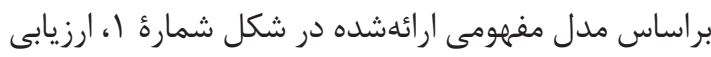

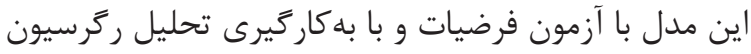

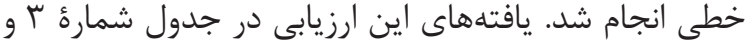

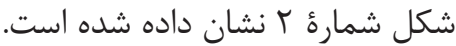

براساس نتايج به دست آمده در جدول شمارئ آ، سطح

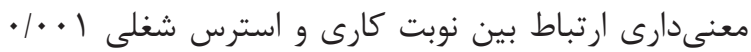

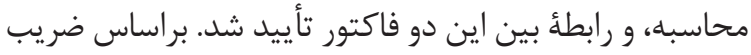

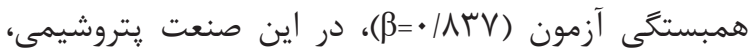

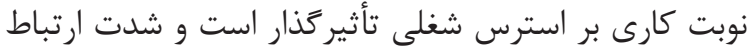

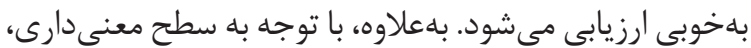

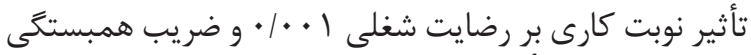

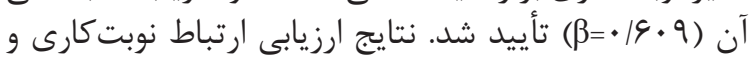

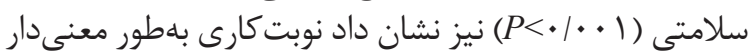

جدول ا. يافتههاى فردى و دموكرافيك

\begin{tabular}{|c|c|c|}
\hline مقادير & \multicolumn{2}{|c|}{ متغير } \\
\hline$r N / 1 r \pm \Delta / / F$ & \multicolumn{2}{|c|}{ سن (سال) } \\
\hline$\Lambda / \Delta r \pm r / \Lambda r$ & \multicolumn{2}{|c|}{ سابقهُ كار (سال) } \\
\hline$\Delta V(\% \backslash \Delta / \wedge)$ & زن ا ت ا & \multirow{2}{*}{ جنسيت } \\
\hline$r \cdot r(\% \wedge F / T)$ & مرد & \\
\hline $10(\% / r)$ & ديِله & \multirow{4}{*}{ تحصيلات } \\
\hline$\Delta V(\% \backslash \Delta / \wedge)$ & فوقدييلم & \\
\hline$r / D(\% \Delta 9 / V)$ & ليسانس و بالاتر & \\
\hline$V r(\% \cdot / r)$ & فوقليسانس و بالاتر & \\
\hline
\end{tabular}

جدول r. نتايج آزمون كولموكروف-اسميرنوف

\begin{tabular}{|c|c|c|c|}
\hline وضعيت نرمال & $P$-Value & آمارة آزمون & فاكتور \\
\hline تأييد & .119 & $.1 .9 V$ & نوبت كارى \\
\hline تأييد & $\cdot / \cdot \wedge \Lambda$ & . $/ 499$ & استرس شغلى \\
\hline تأييد & .1 .91 & $\cdot / T V D$ & رضايت شغلى \\
\hline تأييد & $\cdot / \cdot \vee \Delta$ & .1945 & سلامتى \\
\hline
\end{tabular}

جدول r. نتايج ارزيابى مدل مفهومى

\begin{tabular}{|c|c|c|c|c|c|c|}
\hline \multirow{2}{*}{$P$} & \multirow{2}{*}{$\mathbf{t}$} & \multirow{2}{*}{ ضرايب استاندارد } & \multicolumn{2}{|c|}{ ضرايب غيراستاندارد } & \multirow{2}{*}{ فاكتور وابسته } & \multirow{2}{*}{ فاكتور مستقل } \\
\hline & & & خطاى استاندارد & B & & \\
\hline$\cdot 1 \cdot \cdot 1$ & TN/ANG & - /ArV & ( ) & $\cdot 1190$ & استرس شغلى & نوبت كارى \\
\hline$\cdot 1 \cdot \cdot 1$ & IF/DTA & $\cdot 19 \cdot 9$ & .1 .49 & .1949 & رضايت شغلى & نوبت كارى \\
\hline$\cdot 1 \cdot \cdot 1$ & Q/TFV & . /prq & $\cdot / \cdot r q$ & • MGr & سلامتى & نوبت كارى \\
\hline$\cdot 1 \cdot .1$ & $\mid f / T \cdot F$ & $\cdot 19 \cdot r$ & $\cdot|\cdot|+\mid$ & $\cdot|\Delta \wedge\rangle$ & استرس شغلى & رضايت شغلى \\
\hline$\cdot 1 \cdot .1$ & 19/9दर & - NTS &.$/ . \mathrm{TV}$ & - IDFE & سلامتى & رضايت شغلى \\
\hline$\cdot 1 \cdot \cdot 1$ & V/VAr & $\cdot / \mu \wedge \cdot$ & $\cdot / \cdot r \Lambda$ & $\cdot / r q 4$ & سلامتى & استرس شغلى \\
\hline
\end{tabular}




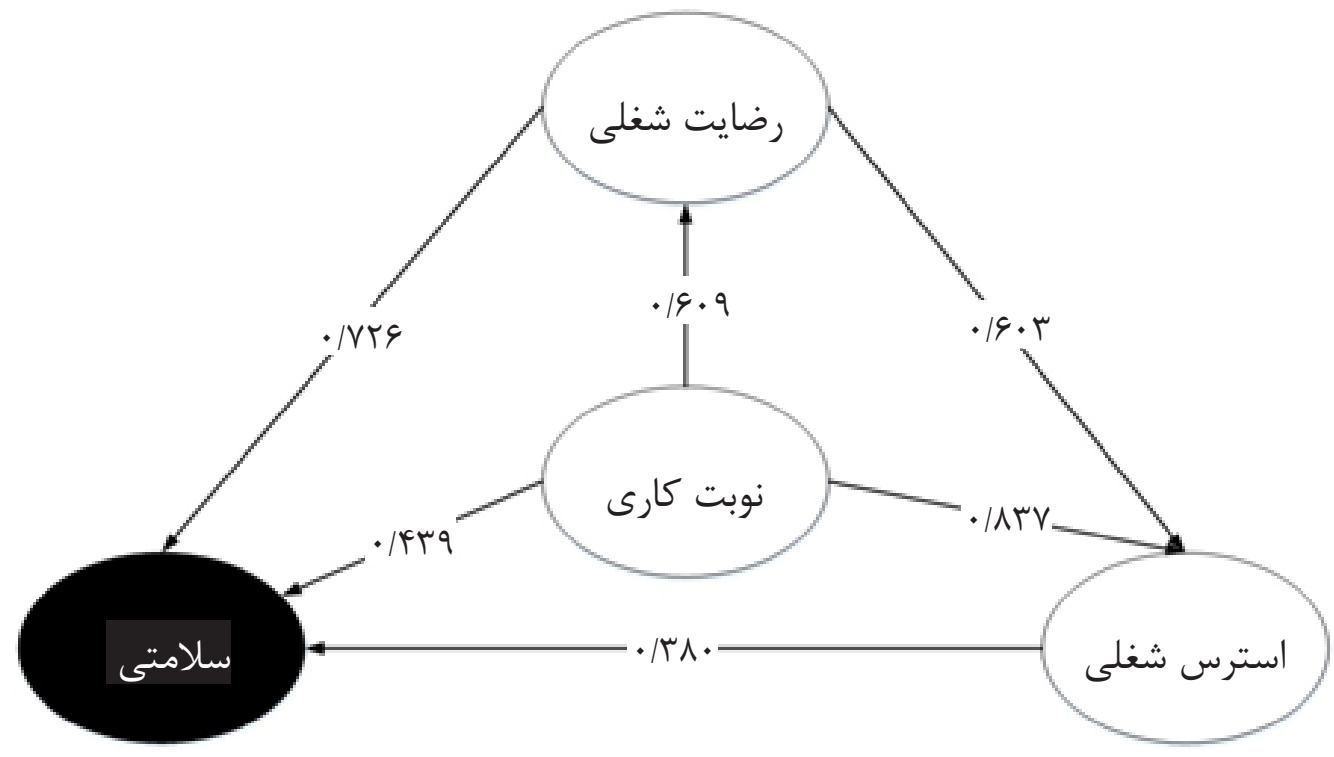

شكل r. مدل نهايبى مطالعه

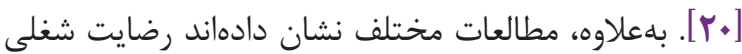

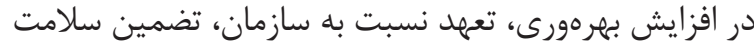

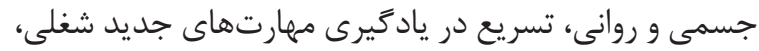

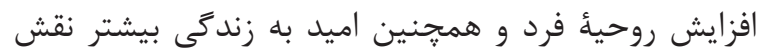

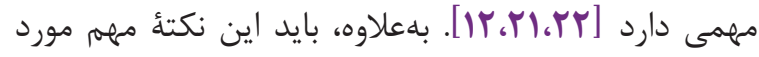

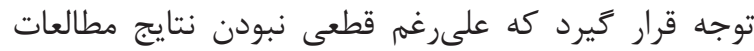

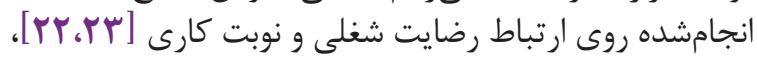

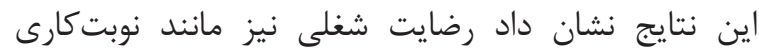

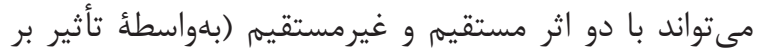
استرس شغلى) بر سلامتى تأثير كذار باشد.

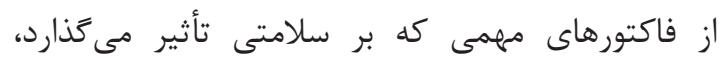

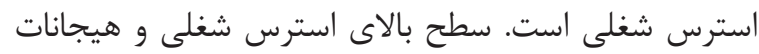

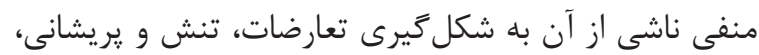

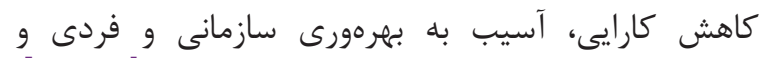

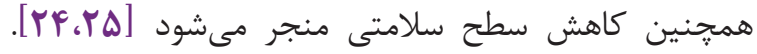

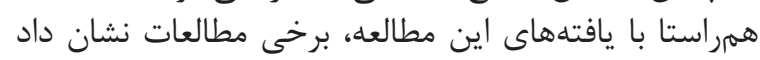

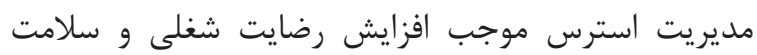

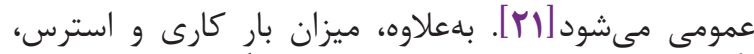

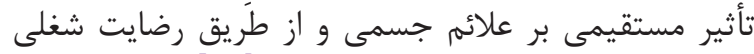

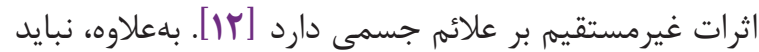

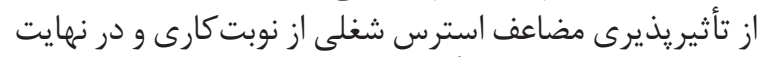

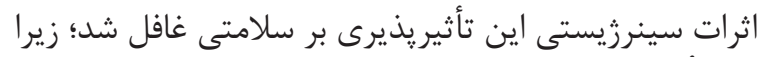

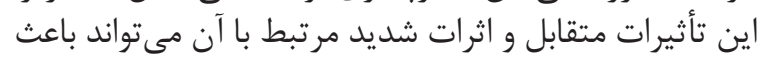

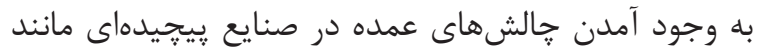

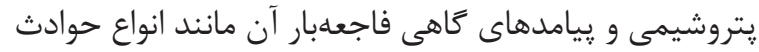

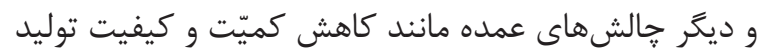

و بهرهورى شود [rع]

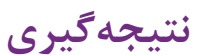

يافتهاى مطالعأه حاضر بار ديكر به اهميت موضوع

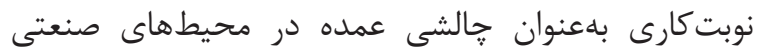

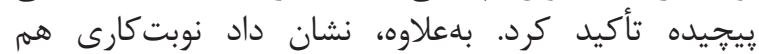

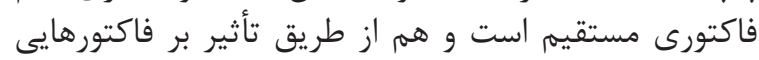

نتايج مطالعه نشان داد كار در نظام نوبت كارى، فاكتور

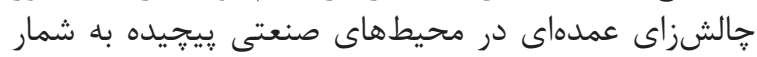

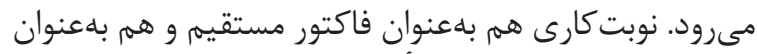

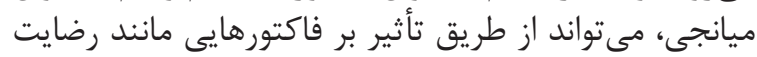

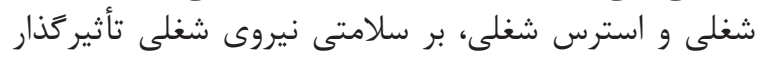

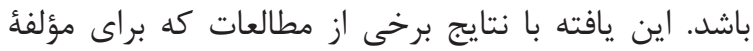

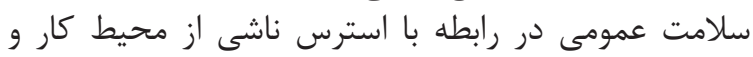

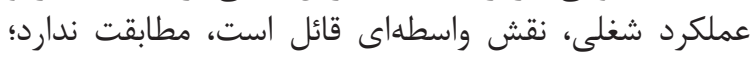

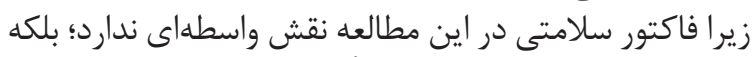

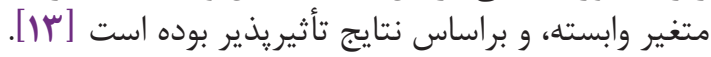
براساس مدل ارائهشه در اين مطالعه و همجنين

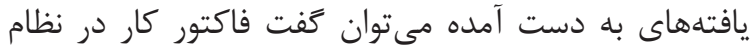

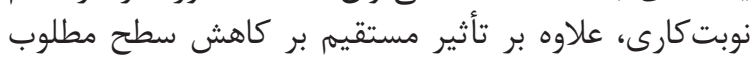

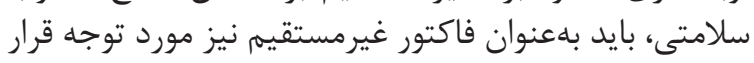

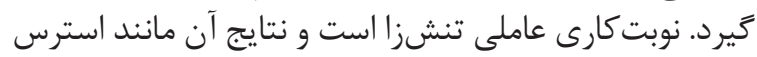

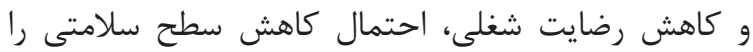

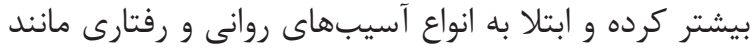

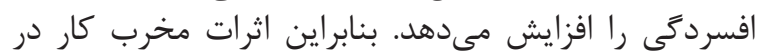

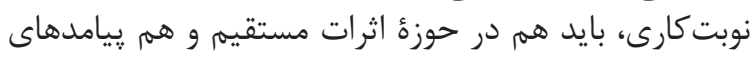

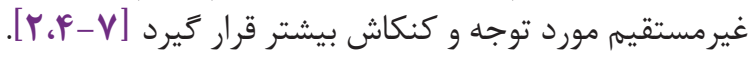

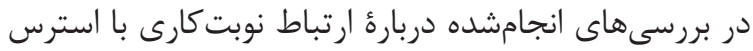

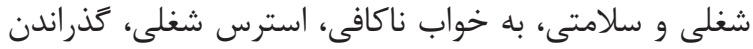

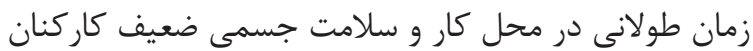

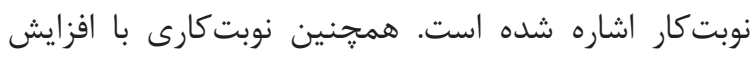

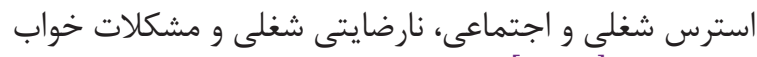

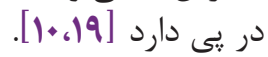
يكى از يافتههاى مهمه اين مطالعه تأثيريذيرى رضايت

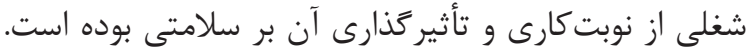

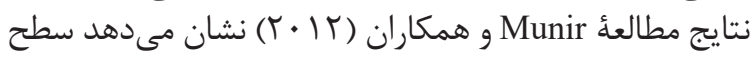

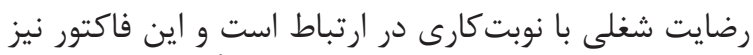

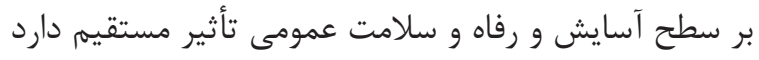




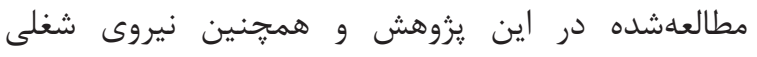

$$
\begin{aligned}
& \text { مشاركت كننده تشكر و قدردانى مى كنيند. } \\
& \text { تعارض منافع } \\
& \text { در اين مطالعه هيجزَّنه تضاد منافعى وجود ندارد. }
\end{aligned}
$$

\section{References}

1. Berger AM, Hobbs BB. Impact of shift work on the health and safety of nurses and patients. Clinical journal of oncology nursing. 2006;10(4). https://doi.org/10.1188/06.CJON.465-471

2. Choobineh A, Soltanzadeh A, Tabatabaee H, Jahangiri M, Neghab M, Khavaji S. Shift work-related psychosocial problems in 12-hour shift schedules of petrochemical industries. International Journal of Occupational Hygiene. 2011;3(1):3842.

3. Pan A, Schernhammer ES, Sun Q, Hu FB. Rotating night shift work and risk of type 2 diabetes: two prospective cohort studies in women. PLoS medicine. 2011;8(12):e1001141. https://doi.org/10.1371/journal.pmed.1001141 PMID:22162955 PMCID:PMC3232220

4. Choobineh A, Soltanzadeh A, Tabatabaee H, Jahangiri $\mathrm{M}$, Khavaji S. Health effects associated with shift work in 12-hour shift schedule among Iranian petrochemical employees. International journal of occupational safety and ergonomics. 2012;18(3):419-27.https://doi.org/10.1080/1080 3548.2012.11076937 PMID:22995139

5. Knutsson A. Health disorders of shift workers. Occupational medicine. 2003;53(2):1038.https://doi.org/10.1093/occmed/kqg048 PMID: 12637594

6. Choobineh A, Soltanzadeh A, Tabatabaee S, Jahangiri M, Khavaji S. Shift work-related health problems in" 12-hour shift" schedule in petrochemical industries. Iran Occupational Health. 2010;7(1):44-53.

7. Choobineh A, Soltanzadeh A, TabatabaeeS, JAhangiri M. Investigating Health Problems and Their Associated Risk Factors among Employees of Iranian Petrochemical Industries with Emphasis on Shift Working. International Journal of Occupational Hygiene. 2015;7(2):61-8.
مانند رضايت شغلى و استرس شغلى، بهعنوان ميانجى و

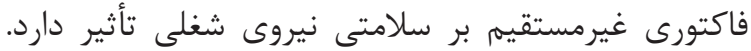

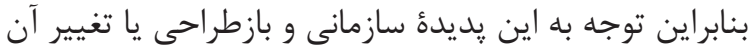

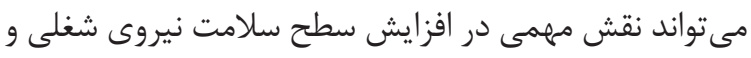
انخيزش شغلى و بهروزورى سازمانى داشته بـ باشد.

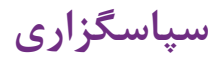

محققان اين مطالعه از مجموعله صنعت يتروشيمى

8. Faragher EB, Cass M, Cooper CL. The relationship between job satisfaction and health: a meta-analysis. InFrom Stress to Wellbeing Volume 12013 (pp. 254-271). Palgrave Macmillan, London.

9. Conway PM, Campanini P, Sartori S, Dotti R, Costa G. Main and interactive effects of shiftwork, age and work stress on health in an Italian sample of healthcare workers. Applied ergonomics. 2008;39(5):630-9. https://doi.org/10.1016/j. apergo.2008.01.007 PMID: $\underline{18313640}$

10. Gerber M, Hartmann T, Brand S, Holsboer-Trachsler E, Pühse U. The relationship between shift work, perceived stress, sleep and health in Swiss police officers. Journal of Criminal Justice. 2010;38(6):1167-75.https://doi. org/10.1016/j.jcrimjus.2010.09.005

11. Dagget T, Molla A, Belachew T. Job related stress among nurses working in Jimma Zone public hospitals, South West Ethiopia: a cross sectional study. BMC nursing. 2016;15(1):39. https://doi.org/10.1186/s12912-016-0158-2 PMID:27313502 PMCID:PMC4910212

12. Sveinsdóttir H, Biering P, Ramel A. Occupational stress, job satisfaction, and working environment among Icelandic nurses: a cross-sectional questionnaire survey. International journal of nursing studies. 2006;43(7):875-89. https://doi.org/10.1016/j.ijnurstu.2005.11.002 PMID:16360157

13. Vosoughi NA, Rohollahi A, Mohamad HH. The effect of job stress on general health and job performance on air traffic controllers (atc). 2016.

14. Orchard CA. Persistent isolationist or collaborator? The nurse's role in interprofessional collaborative practice. Journal of Nursing Management. 2010;18(3):248-57.https://doi.org/10.1111/ j.1365-2834.2010.01072.x PMID: 20546464 
15. Rameshbabu A, Reddy DM, Fleming R. Correlates of negative physical health in call center shift workers. Applied Ergonomics. 2013;44(3):350-4.https://doi.org/10.1016/j.apergo.2012.09.002 PMID:23040668

16. Ahghar G. The role of school organizational climate in occupational stress among secondary school teachers in Tehran. International Journal of Occupational Medicine and Environmental Health. 2008;21(4):319-29. PMID: 19228578

17. Smith PC. The measurement of satisfaction in work and retirement: A strategy for the study of attitudes. 1969.

18. Nazifi M, Mokarami H, Akbaritabar A, Faraji Kujerdi M, Tabrizi R, Rahi A. Reliability, Validity and Factor Structure of the Persian Translation of General Health Questionnaire (GHQ-28) in Hospitals of Kerman University of Medical Sciences. Journal of Fasa University of Medical Sciences/Majallah-i Danishgah-i Ulum-i Pizishki-i Fasa. 2014;3(4).

19. Ma CC, Andrew ME, Fekedulegn D, Gu JK, Hartley TA, Charles LE, et al. Shift work and occupational stress in police officers. Safety and health at work. 2015;6(1):25-9. https://doi.org/10.1016/j. shaw.2014.10.001 PMID:25830066

20. Munir F, Nielsen K, Garde AH, Albertsen K, Carneiro IG. Mediating the effects of work-life conflict between transformational leadership and health-care workers' job satisfaction and psychological wellbeing. Journal of nursing management. 2012;20(4):512-21.https://doi.org/10.1111/ j.1365-2834.2011.01308.x PMID:22591153
21. Antoniou A-SG, Davidson MJ, Cooper CL. Occupational stress, job satisfaction and health state in male and female junior hospital doctors in Greece. Journal of managerial psychology. 2003;18(6):592-621. https://doi. org/10.1108/02683940310494403

22. Takahashi M, Nakata A, Haratani T, Otsuka Y, Kaida K, Fukasawa K. Psychosocial work characteristics predicting daytime sleepiness in day and shift workers. Chronobiology international. 2006;23(6):1409-22. https://doi.org/10.1080/07420520601100963 PMID: 17190723

23. Sveinsdottir H. Self-assessed quality of sleep, occupational health, working environment, illness experience and job satisfaction of female nurses working different combination of shifts. Scandinavian journal of caring sciences. 2006;20(2):229-37. https://doi.org/10.1111/ j.1471-6712.2006.00402.x PMID: 16756530

24. Noblet A, LaMontagne AD. The role of workplace health promotion in addressing job stress. Health promotion international. 2006;21(4):34653. https://doi.org/10.1093/heapro/dal029 PMID: 16880197

25. Peiman PF, Mansour L, Sadeghi M, Purebraham $\mathrm{T}$. The relationship of job stress with marital satisfaction and mental health in nurses of Tehran hospitals. 2013.

26. Culpepper L. The social and economic burden of shift-work disorder. Journal of Family practice. 2010;59(1):S3-S. 